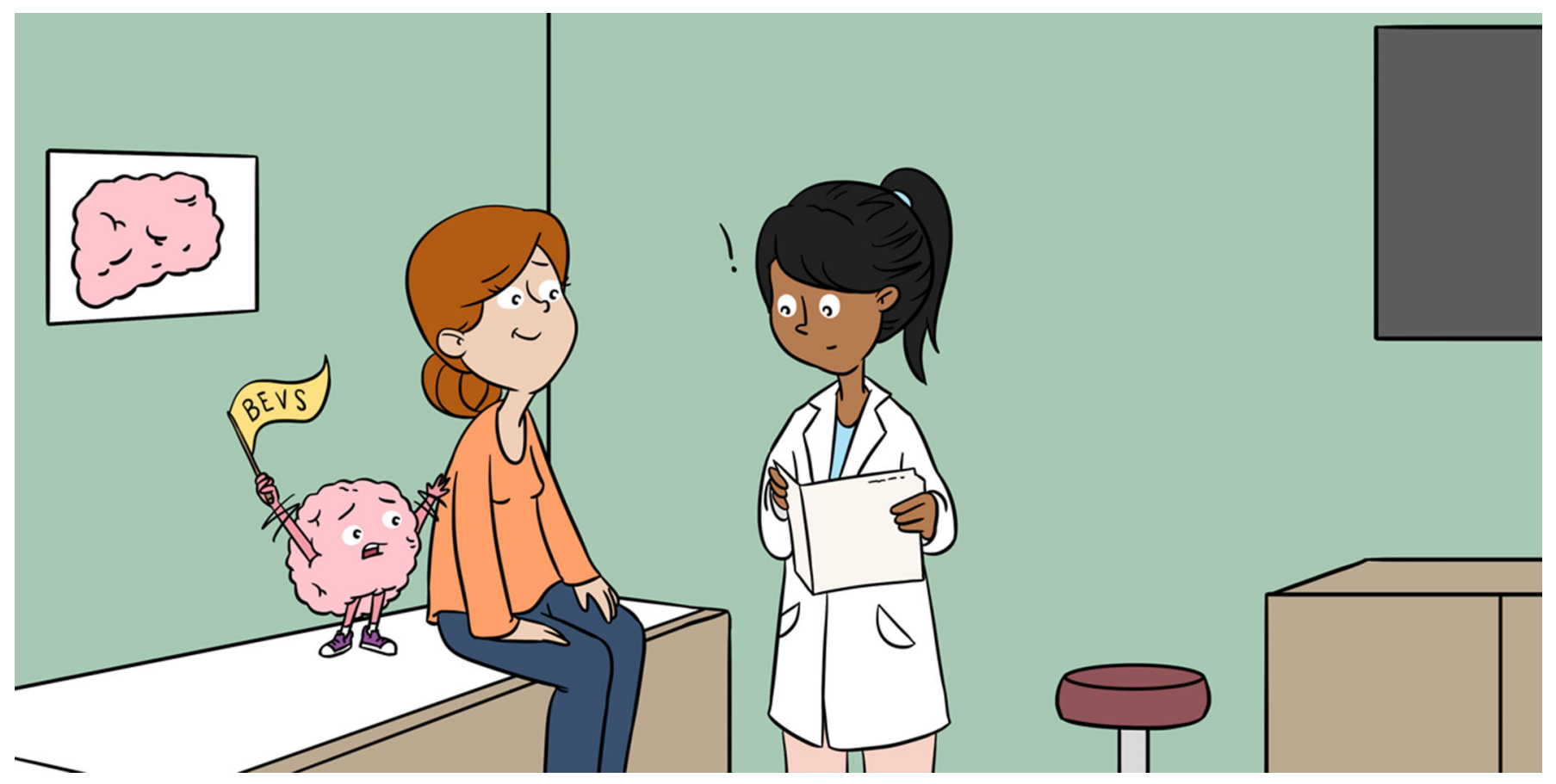

\title{
HOW YOUR BLOOD KNOWS YOUR BRAIN IS SICK
}

\author{
Sabrina Loudjani ${ }^{1}$, Sridar Narayanan ${ }^{2}$, Arsalan S. Haqqani ${ }^{3}$ and AmanPreet Badhwar ${ }^{1 *}$ \\ ${ }^{1}$ Centre de Recherche de l'Institut Universitaire de Gériatrie de Montréal, Université de Montréal, Montreal, QC, Canada \\ ${ }^{2}$ McConnell Brain Imaging Centre, Montreal Neurological Institute, McGill University, Montreal, QC, Canada \\ ${ }^{3}$ Human Health Therapeutics Research Centre, National Research Council, Ottawa, ON, Canada
}

YOUNG REVIEWERS:

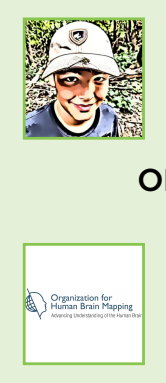

ADRIAN

AGE: 10

ORGANIZATION

FOR

HUMAN

BRAIN

MAPPING

AGES: 8-15
Alzheimer's disease (AD) is a complex disease that attacks the brain that mostly affects people 65 years and older. AD affects more and more people each year. A major problem with $A D$ is that it is diagnosed too late. A big goal is to find ways to help doctors identify the disease early, so they can better help AD patients. Biomarkers are something that can tell you if a part of the body is feeling healthy or is being attacked by a disease. This article will describe one exciting new category of biomarkers that carry information from the brain into the blood. These biomarkers can be used to see how healthy the brain is feeling or if it is getting hurt by a disease like AD.

\section{WHAT IS ALZHEIMER'S DISEASE?}

Alzheimer's disease (AD) is a devastating neurodegenerative disease that is usually diagnosed in people 65 years and older. The word neurodegenerative means that the disease affects the nervous system, and that, over time, the disease damages and breaks down (or 


\section{NEURON}

A special kind of brain cell that help carry out functions like learning and memory.

\section{AMYLOID-BETA}

A protein that is increased in Alzheimer's disease.

\section{Tau}

A protein that is increased in Alzheimer's disease

\section{BIOMARKER}

Biomarkers can be anything (like a protein found in the blood) that tells you if a part of the body is feeling healthy or is being attacked by a disease.

\section{CSF}

Cerebrospinal fluid (CSF), a fluid that the brain and spinal cord are bathed in. degenerates) nerve cells, called neurons. In particular, AD damages the brain neurons that control our bodies and determine how we think, remember, and feel. Multiple factors contribute to the risk of getting $A D$ and how fast it gets worse [1], including older age, buildup of certain toxic proteins in the brain called amyloid-beta and tau, and a person's genes. Currently there is no cure for AD, but there are a few drugs that can temporarily reduce symptoms in people who have it.

$A D$ is a disease that starts long before it is diagnosed, lasts for many years, and worsens over time. In its earliest stages, the person displays no memory-loss symptoms, but the brain is starting to get damaged. For a while, the brain can keep performing its functions by having the healthy parts help the damaged parts. As damage increases, the brain is less able to work, and the person starts having some memory problems. In the most severe stage of $A D$, known as dementia, people experience severe memory loss, confusion (like confusing the past with the present), difficulty completing everyday tasks (like getting dressed or even swallowing), and difficulty speaking.

By the time a person is diagnosed with $A D$, the person's brain has already suffered quite a bit of damage, which is extremely hard to repair with medication. Doctors need a way to test for $A D$ even before noticeable symptoms appear, or when those symptoms are still mild. That is why AD biomarkers, which will be described below, are a great research topic, since they allow doctors and scientists to detect AD in its earlier stages, which could help thousands of people.

\section{WHAT IS A DISEASE BIOMARKER?}

A disease biomarker can be anything-from a protein found someplace in the body, for example in the blood, to a picture of the brain taken using brain-imaging techniques. A biomarker is something that can tell you if a part of the body is feeling healthy or is being attacked by a disease. For example, a doctor can check the amount of a sugar called glucose in a sample of your blood to see if you have diabetes. In this case, a high sugar level in the blood is a biomarker for diabetes. For complex diseases like AD, doctors and scientists rely heavily on biomarkers for identifying people in the early stages, when symptoms are mild or absent.

\section{Well-Accepted Biomarkers of AD}

A true diagnosis of AD can only be confirmed by checking the brain after the patient has died. Specifically, doctors check to see if there are increased amounts of two proteins in the brain: amyloid-beta and tau. Thanks to technological advances, increases in these two proteins can now be detected in a living person, using two well-accepted methods [2]. The levels of the proteins can be measured in the cerebrospinal fluid (CSF), a fluid that the brain and spinal cord are bathed in, or a 
Figure 1

Getting and analyzing CSF. To get a CSF sample, a needle is inserted into the lower back, known as the lumbar area of the spinal cord. Scientists can study the proteins in the CSF sample. In $A D$, the quantities of amyloid-beta and tau proteins in the CSF are used as biomarkers.

\section{PET}

Positron emission topography (PET) is used to identify protein amounts in the brain of a living person.

BEVs

Brain-secreted extracellular vesicles (BEV) are little packages that carry and deliver messages, in the form of biological molecules, throughout the body.

\section{BLOOD-BRAIN}

\section{BARRIER}

A line of bodyguards that protect our brain from harmful substances

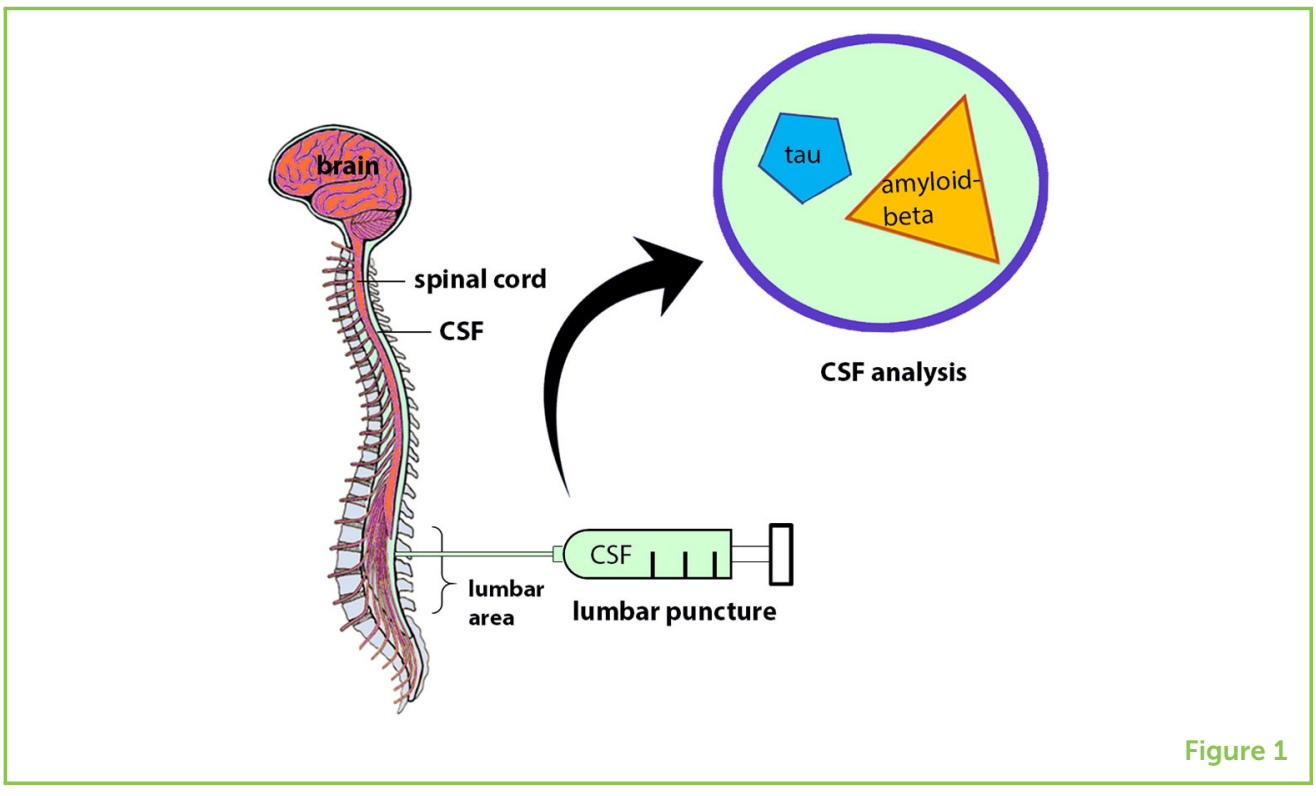

brain imaging technique called positron emission tomography (PET) can be used to detect the buildup of the proteins in the brain.

\section{WHY DO WE NEED BLOOD BIOMARKERS FOR AD?}

Getting the CSF to check for the levels of amyloid-beta and tau requires inserting a needle into the spine (Figure 1 ). This process, called a lumbar puncture, is unpleasant for most people. PET imaging involves injecting radioactive tracer substances into an arm vein. The substances light up amyloid-beta or tau in the brain, depending on the tracer injected, which allows the PET scanner to take a picture of these proteins in the brain (Figure 2). While PET imaging is not as unpleasant as CSF collection, it is very expensive and not available at most medical centers.

Scientists are trying to find blood-based biomarkers for AD. A blood-based test will hurt less than the CSF test, and will be cheaper and more available than PET imaging [3]. Blood-based biomarkers for AD will allow doctors to test people more easily and more often, and to detect AD earlier. Scientists and doctors believe that if drugs are given before the brain gets too damaged, they might have a better chance at slowing $A D$.

\section{AN ATTRACTIVE NEW BLOOD BIOMARKER FOR AD}

Currently, scientists are studying a few different kinds of AD biomarkers in blood. Of these, we will focus on brain-secreted extracellular vesicles (BEVs) [4]. Since the brain is such an essential organ and it cannot repair itself as well as some other body parts, it is heavily protected by line of "bodyguards" called the blood-brain barrier (BBB). 
Figure 2

PET imaging. PET imaging is used to identify protein amounts in the brain of a living person. The patient lies down on the PET scanner table, which can move in and out of the machine. First, a radioactive tracer (purple) is injected into the patient's arm. The radioactivity allows the machine to find and track the tracer. The tracer then travels to the brain and binds to the specific protein it is designed for, in this case amyloid-beta. The imaging machine locates it and takes a picture: brighter and more red means more protein (PET images source: https:// radiology.ucsf. edu/patient-care/ services/ specialtyimaging/alzheimer)

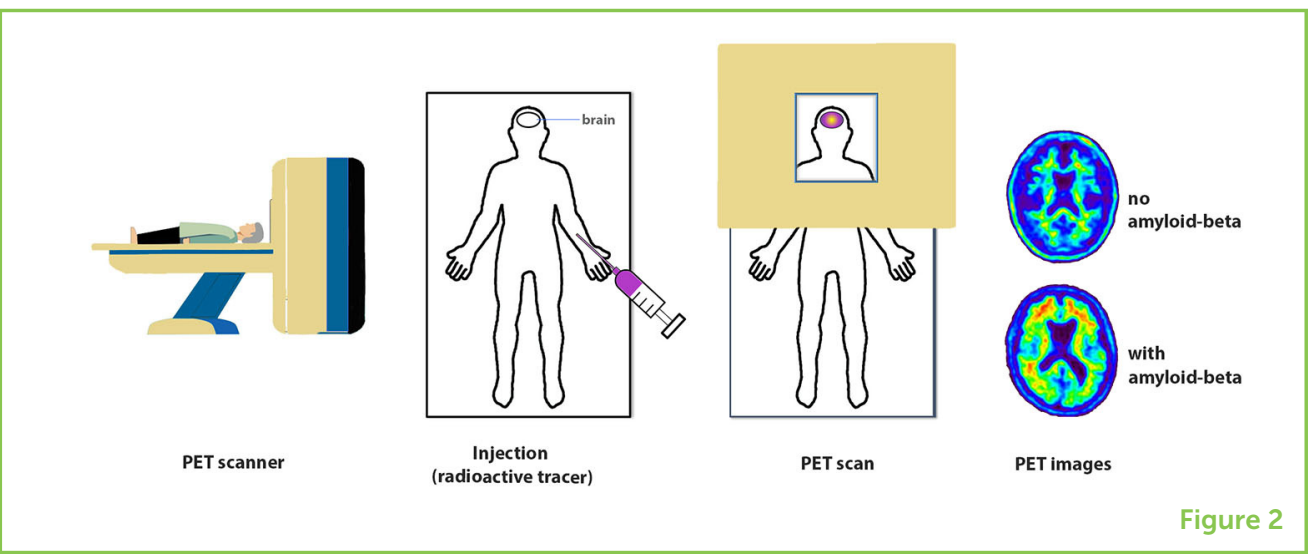

The BBB allows only the right things to enter or exit the brain based on their chemical signature, kind of like a passport. BEVs are some of the few structures that are allowed to pass through the BBB.

So, what are BEVs? In general, vesicles are like little packages that carry and deliver messages, in the form of biological molecules, throughout the body. An extracellular vesicle is a vesicle that is released from a cell and carries messages from that cell to other cells. So, BEVs are vesicles that are released by brain cells, and they carry messages from the brain, across the BBB, to the rest of the body. Scientists can successfully isolate BEVs from the blood and analyze their contents, to see if they are carrying increased amounts of amyloid-beta and tau proteins. This is cool, because BEVs can tell us what is going on directly in the brain, from just a blood sample! To date, AD scientists have studied BEVs released into the blood by four different kinds of brain cells-neurons, neural precursors, astrocytes, and vascular cells that make up the brain's blood vessels [4] (Figure 3). Since BEVs released in blood by neurons have been the most looked at, we provide some facts below of these findings [4].

\section{BEVs RELEASED BY NEURONS}

To establish BEVs as a good blood biomarker for AD, scientists first need to determine whether more amyloid-beta and/or tau proteins are found in BEVs from people with AD than in BEVs of people without $A D$. By studying BEVs from neurons, scientists have found that there is more amyloid-beta in AD patients than in people without $A D$, and the amount increases as AD gets worse. This shows that the amount of amyloid-beta in BEVs is related to the severity of the disease, which is an important characteristic of a good biomarker. Also, amyloid-beta in BEVs was found in AD patients up to 10 years before their diagnosis, which means scientists may be able to use these BEVs to determine who has $A D$, even before patients have any symptoms. Tau in BEVs has also been detected in people with and without AD, but studies are 
Figure 3

Brain-secreted extracellular vesicles. Vesicles sent from the brain to the rest of the body are known as BEVs. BEVs carry information about the brain's health. So, if the brain is sick, BEVs could let us know before a patient's symptoms start getting serious. On the left is a BEV being released from the brain into the blood. BEVs can contain various molecules, such as proteins. Currently, scientists have studied $B E V s$ released by four different kinds of brain cells in

blood-neurons, neural precursors, astrocytes, and vascular cells.

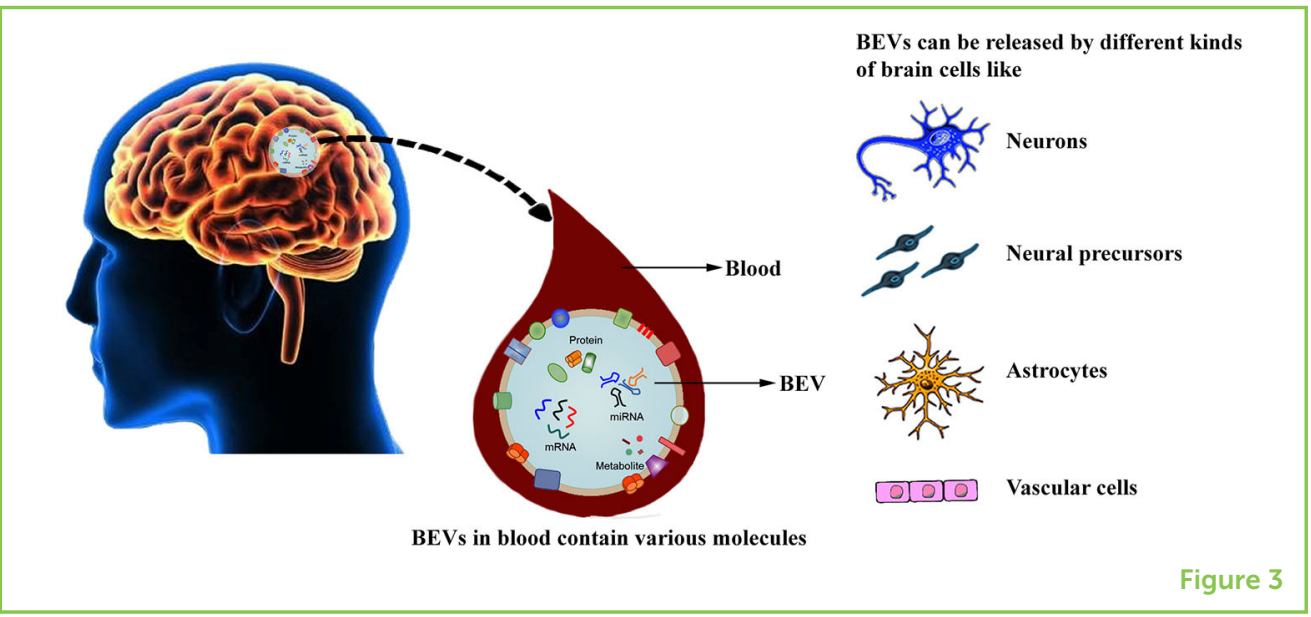

still unclear on whether tau is increased in the BEVs of AD patients, so more work needs to be done.

For those that are curious, since AD can be caused by multiple factors, scientists have started looking at other brain proteins contained in BEVs for their biomarker potential, including neuronal proteins (from neurons). While BEV biomarkers are looking promising more studies are needed to know their best use for AD research.

\section{A MORE COMPLETE PICTURE}

$A D$ is like a puzzle: multiple pieces must be put together to see the big picture. Scientists have not found all the pieces yet. However, combining the information we have can bring us closer to understanding this disease. BEVs are one piece of this puzzle. Perhaps analyzing biomarkers like BEVs will be an early, easy test used to initially determine if a person is starting to develop AD. Then, doctors can move on to more elaborate tests like PET and CSF. Together, these methods will bring us closer to understanding $A D$ and helping the many patients who struggle with this terrible disease.

\section{ACKNOWLEDGMENT}

We would like to thank Dr. Désirée Lussier and Noah Canfield in helping to make the manuscript more accessible to young curious readers.

\section{REFERENCES}

1. Badhwar, A., McFall, G. P., Sapkota, S., Black, S. E., Chertkow, H., Duchesne, S., et al. 2019. A multiomics approach to heterogeneity in Alzheimer's disease: focused review and roadmap. Brain 143:1315-31. doi: 10.1093/brain/awz384 
2. Jack, C. R. Jr., Bennett, D. A., Blennow, K., Carrillo, M. C., Dunn, B., Haeberlein, S. B., et al. 2018. NIA-AA research framework: toward a biological definition of Alzheimer's disease. Alzheimers Dement. 14:535-62. doi: 10.1016/j. jalz.2018.02.018

3. Hampel, H., O’Bryant, S. E., Molinuevo, J. L., Zetterberg, H., Masters, C. L., Lista, S., et al. 2018. Blood-based biomarkers for Alzheimer disease: mapping the road to the clinic. Nat. Rev. Neurol. 14:639-52. doi: 10.1038/s41582-018-0079-7

4. Badhwar, A., and Haqqani, A. S. 2020. Biomarker potential of brain-secreted extracellular vesicles in blood in Alzheimer's disease. Alzheimers Dement. (Amst). 12:e12001. doi: 10.1002/dad2.12001

SUBMITTED: 12 May 2020; ACCEPTED: 12 November 2020; PUBLISHED ONLINE: 08 December 2020.

EDITED BY: Julia W. Y. Kam, University of Calgary, Canada

CITATION: Loudjani S, Narayanan S, Haqqani AS and Badhwar A (2020) How Your Blood Knows Your Brain Is Sick. Front. Young Minds 8:561561. doi: 10.3389/frym. 2020.561561

CONFLICT OF INTEREST: The authors declare that the research was conducted in the absence of any commercial or financial relationships that could be construed as a potential conflict of interest.

COPYRIGHT (C) 2020 Loudjani, Narayanan, Haqqani and Badhwar. This is an open-access article distributed under the terms of the Creative Commons Attribution License (CC BY). The use, distribution or reproduction in other forums is permitted, provided the original author(s) and the copyright owner(s) are credited and that the original publication in this journal is cited, in accordance with accepted academic practice. No use, distribution or reproduction is permitted which does not comply with these terms.

\section{YOUNG REVIEWERS}

\section{ADRIAN, AGE: 10}

My name is Adrian, I have been into history for 2-3 years, my favorite school subjects are History and Math. I like reading very much, and I am in 5th grade.

\section{ORGANIZATION FOR HUMAN BRAIN MAPPING, AGES: 8-15}

As part of the Kids Live Review Event at OHBM 2020, Adrian, Louie, Pinaki, Reina, and Tian, grilled the scientists on their work in front of an audience. This group of elite reviewers provided pointed feedback that improved the quality of each of these papers, ranging from boredom, irritability, and social learning, to brain surgeries and Alzheimer's disease. 

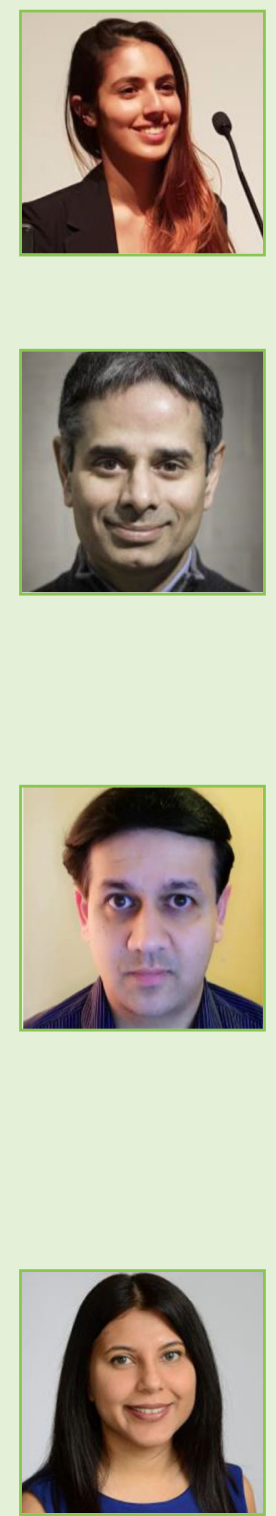

\section{AUTHORS}

\section{SABRINA LOUDJANI}

Sabrina Loudjani is an undergraduate student at the University of Montreal, doing a major in biomedical sciences. She is interested in conducting research on brain diseases, such as Alzheimer's disease. Since 2018, Sabrina has conducted multiple internships under the supervision of Dr. AmanPreet Badhwar. With great passion and enthusiasm, she hopes to continue growing as a scientist in this field.

\section{SRIDAR NARAYANAN}

Dr. Sridar Narayanan obtained a Ph.D. in Neuroscience from McGill University. He is now an Assistant Professor in the Department of Neurology and Neurosurgery at McGill University, and is a scientist in the McConnell Brain Imaging Centre of the Montreal Neurological Institute. His main research is on developing new ways to scan the brain, and on how to extract as much information as possible from brain images to help study neurological diseases, such as multiple sclerosis and Alzheimer's disease.

\section{ARSALAN S. HAQQANI}

Dr. Arsalan S. Haqqani completed his Ph.D. from the University of Ottawa in the Department of Biochemistry, Microbiology, and Immunology in the area of cancer research, under the supervision of Dr. H. C. Birnboim. In 2002, he joined the National Research Council of Canada (NRC) as a post-doctoral fellow in the laboratories of Dr. Danica B. Stanimirovic and Dr. John F. Kelly to study biomarkers in brain diseases. He is currently a Senior Research Officer at NRC, leading a project to identify brain-specific molecules that can be used to create new treatments for brain diseases.

\section{AMANPREET BADHWAR}

Dr. AmanPreet Badhwar grew up in India, where she loved climbing mango trees and caring for monkeys. She came to Canada as a teenager and went on to get her Ph.D. from McGill University. She is currently an Assistant Professor in the Department of Pharmacology and Physiology at the University of Montreal in Montreal, Canada. Dr. Badhwar combines different methods to better understand Alzheimer's disease. These methods vary from looking at the entire brain using imaging to looking at small molecules that make up the brain. Dr. Badhwar also explains science using her paintings. *amanpreet.badhwar@criugm.qc.ca 